\title{
Automated Screening System for Covid Safety
}

\author{
Yarram Sai Subhash Reddy, Sri Krishna Borra, Koye Sai Vishnu Vamsi, Nandipati Jaswanth Sai, Koye \\ Jayanth
}

\begin{abstract}
COVID-19 is a life-threatening virus taking the lives of thousands of people every day throughout the world. Even though many organizations and companies worked hard and developed vaccines, production of vaccines at large scale to meet today's demand is not an easy job as there is a shortage of raw materials and cases are rising steeply. Inoculation of every individual cannot be achieved in the foreseeable future. Even the government is vaccinating people in a phased manner prioritizing older people and people who are more vulnerable to the virus. The main objective of this work is to provide an optimum solution for COVID-19 indoor safety for industries, offices, and commercial places where footfall is high. This work focus on automation of temperature sensing and mask detection which is usually carried out by a person. Elimination of human intervention reduces the risk of contraction and spreading and avoids mistakes due to human negligence. Continuous monitoring of a person is not possible and there is no guarantee that a person who is entering a place wearing a mask puts it on until he leaves it. This research intends to implement mask detection along with surveillance which is cost effective as it does not require additional hardware setup.
\end{abstract}

Keywords: Arduino Uno, Computer Vision, COVID-19, Deep Learning, Convolutional Neural Networks

\section{INTRODUCTION}

$T_{\text {he occurrence of novel infectious respiratory disease }}$ COVID-19 caused by coronavirus (SARS-Cov-2 virus) has had a detrimental effect on every aspect of people's lives globally since the beginning of the year 2020. On March 11th of 2020, the World Health Organization declared that COVID-19 as a global pandemic, acknowledging significant global spread of an infectious disease[1]. China had been the first country with a widespread outbreak in January, and South Korea, Iran and Italy following in February with their own outbreaks. Soon, the virus was in all continents and over 177 countries, and as of this writing, the United States has the highest number of confirmed cases and, sadly, the most deaths. In India, the coronavirus infection number has started to rise again.

Manuscript received on November 15, 2021.

Revised Manuscript received on November 17, 2021.

Manuscript published on December 30, 2021.

*Correspondence Author

Y Sai Subhash Reddy, Department of Computer Science Engineering, Vellore Institute of Technology, Vellore. (Tamil Nadu), India.

Sri Krishna Borra, Department of Electronics and Communication Engineering, National Institute of Technology, Jamshedpur (Jharkhand), India.

Koye Sai Vishnu Vamsi, Department of Computer Science Engineering, Vellore Institute of Technology, Vellore (Tamil Nadu), India.

Nandipati Jaswanth Sai, Department of Computer Science Engineering, Vellore Institute of Technology, Vellore (Tamil Nadu), India.

Koye Jayanth, Department of Computer Science Engineering, Vellore Institute of Technology, Vellore (Tamil Nadu), India.

(C)The Authors. Published by Blue Eyes Intelligence Engineering and Sciences Publication (BEIESP). This is an open access article under the CC BY-NC-ND license (http://creativecommons.org/licenses/by-nc-nd/4.0/)
Previously, it was in its zenith in mid-September and declined steadily until February this year. Now, there is a resurgence in most of the states. Even though the vaccine is available in the global market and India being the largest manufacturer of coronavirus vaccine, inoculation of each and every individual takes a significant time and this goal cannot be achieved in near future. Due to the high population density in India and low availability of raw materials, the government is vaccinating people in a phase manner. It prioritized frontline workers and population above the age of 60 years to be vaccinated first. Then it reduced the cap to 45 years. Once there is an adequate amount of vaccine, the government is planning to reduce the cap further and extend the vaccination drive to every individual of the country.

The second wave of COVID-19 is affecting most of the world and the scenario in India is very grim. Number of cases being registered everyday are increasing rapidly[1]. Even the number of deaths being registered are not on par with that of the first wave. Remdesivir, a drug used to treat coronavirus patients, is not readily available in open markets. So, the risk is still prevalent and the preemptive steps are most necessary now than ever.

The virus is extremely contagious and leads to death in the most vulnerable, particularly those older than 60 and those with underlying conditions. The virus can be transmitted from person to person very easily and the lack of cure and proper medication makes it more deadly[2]. Therefore, the use of face masks and proper sanitization measures have proven to be effective in reducing the spread of the virus. The major and undeniable places of spread include Supermarkets, Grocery stores, Malls, Restaurants, etc. This leads to a major problem as to how to monitor these crowded places.

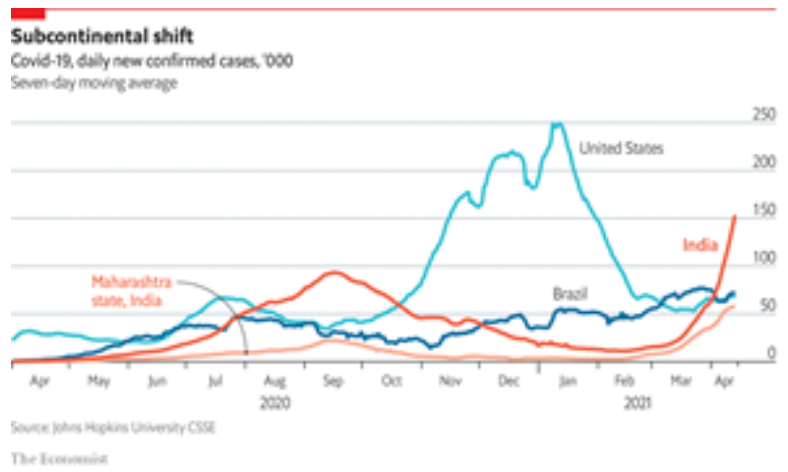

Fig. 1. Graph depicting the rise of COVID cases world wide

This research aims to automate the mandatory security checks such as temperature check and mask detection. These are rudimentary yet essential for reducing the spread of the infectious disease. 


\section{Automated Screening System for Covid Safety}

\section{II.LITERATURE REVIEW}

Face Detection and Recognition Using OpenCV. Maliha Khan; Sudeshna Chakraborty; Rani Astya; Shaveta Khepra. In this paper the work on face detection is done using openCV. Their work has provided us with a good reference in mask detection using openCV. They also mentioned the importance of video and picture detection in today's scenario. Their work has also enabled us in understanding the background process of a face or image detection in the terms of concepts related to PCA and self-space. Automatic Measurement of Human Body Temperature on Thermal Image Using Knowledge-Based Criteria Hurriyatul Fitriyah [1], Aditya Rachmadi [2], Gembong Edhi Setyawan [3] Dept. Informatic/Computer Science, Faculty of Computer Science, University of Brawijaya Veteran Road. 8, 65145 Malang, Indonesia. In this paper they worked on an infrared camera that could be utilized to scan body temperature instantly and non-contact Instead of a thermometer. This paper proposed a non-contact measurement of human body temperature by automatically locating inner-chantus on thermal images. This paper has given us the necessary idea on how the research can implement the contactless temperature measurement in an effective way. Impact of Social Distancing Measures on Coronavirus Disease Healthcare Demand, Central Texas, USA.Xutong Wang1, Remy F. Pasco1, Zhanwei Du1, Michaela Petty, Spencer J. Fox, Alison P. Galvani, Michael Pignone, S. Claiborne Johnston, and Lauren Ancel Meyers. The authors in this publication have presented the need of social distancing and various measures to implement social distancing in an effective way in different areas. Their work has led us to come with the better solution involving the concept of social distancing in the work.

\section{A. Tensorflow:}

\section{III.DEPENDENCIES}

TensorFlow is an open-source and free software library which is created for machine learning. It can be used for a numerous number of tasks but its main focus is on training and inference of deep neural networks. It is a symbolic math library based on dataflow and differential programming

\section{B. Keras:}

Keras is also an open-source software library that provides an interface for python for artificial neural networks. Its main purpose is to act as an interface for the TensorFlow library. Keras is mainly designed to enable fast computation and experimentation with deep neural networks.

\section{NumPy:}

Numerical Python is a library in the python. Its main features are it makes computation and working with large multidimensional arrays and matrices very easy and efficient. It also has a large collection of high-level mathematical functions which are required to work on the arrays and matrices.

\section{Matplotlib:}

Matplotlib is a plotting library which is present in the python programming language. It is a comprehensive library for creating interactive, animated, and static graphs and visualizations in python. It can be used with NumPy for better efficiency.

\section{E. Imutils}

Imutils are a set of functions which are used to make basic image processing functions such as rotation, translation, resizing, skeletonization, and displaying Matplotlib images with open CV easier.

\section{METHODOLOGY}

\section{A. Construction and working:}

This system consists of mainly two units, a computer vision unit using which it can be checked if the person entering is wearing a mask or not, which is implemented using openCV[4] based on raspberry pi and another contactless temperature measuring unit which is used to read the body temperature of the person without physical contact. Both the systems results are integrated in the end to provide the output signal. This whole system is placed at the entrance of the building where it is integrated to a door or a barrier which opens and closes according to the output signal. When a person tries to enter the building, the door is initially closed and when the person steps in front of the system a contactless temperature check is performed using an Arduino Uno which is equipped with an infrared thermometer (MLX90614), after that the next step is to check if the person is wearing a mask or not using computer vision, based on a raspberry pi computer which is equipped with a camera module.

After this, an Arduino board is used which takes input from both the systems (to avoid extra cost and reduce the complexity of the system, the same board can be used which is used in the temperature check unit) and produces an output signal which is used to open the door. The person has to pass both the checks for the doors to open and the person cannot enter into the building if he fails even a single test. A person who fails the test will be asked to go back.

\section{V.COMPUTER VISION UNIT}

\section{A. Dataset:}

To train the mask detection model using deep learning first of all a set of images of people with masks and people without masks are needed, so that the model can learn and improve using this data. For this, images of people with and without masks from various open-source image libraries such as Kaggle, google...etc are collected. The dataset has roughly 1900 images each of people with and without masks.
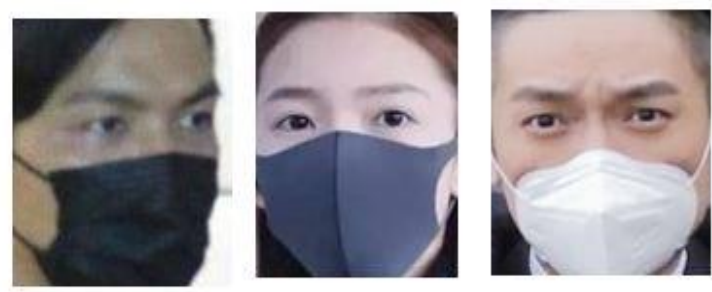

Fig. 2. sample data of people with masks.

Published By:

Blue Eyes Intelligence Engineering and Sciences Publication (BEIESP)

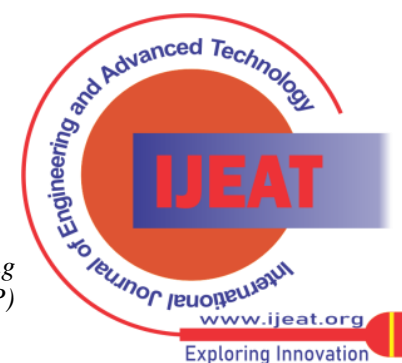



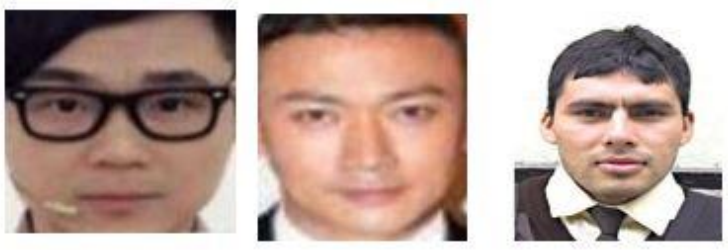

Fig. 3. sample data of people without masks.

\section{Implementation:}

The implementation part of the mask detection system is mainly divided into two parts, the first part is the building and training of the model with the data set and the second part is using the trained model to make predictions on the video stream.

\section{A. Temperature Measurement Unit:}

Temperature measurement system based on Arduino Uno determines the temperature of the commuter. The IR sensor (MLX90614) is equipped for this purpose. Contactless reading is obtained which is then compared with normal human body temperature $\left(37^{\circ} \mathrm{C}\right)$. If the reading obtained exceeds this temperature, he will be asked to go back and the doors remain closed. Otherwise, a positive signal will be sent to the Arduino which is then integrated with the signal obtained from the mask detection unit. IR Temperature Sensor gives the temperature reading (i.e. infrared radiation signal) by transforming it into electrical signal. This electrical signal, which is analog, is measured in voltage. This voltage is converted into corresponding temperature in the Celsius scale. Further, the value is converted to Fahrenheit using the conversion formula.

\section{B. Integration of two units:}

The Temperature Measurement Unit is integrated with the Computer Vision Unit using an Arduino Uno. When the mask detection is performed using the corresponding unit, a digital output is received which is given to Arduino uno as input at digital pin 9[6]. Reading from the temperature measurement unit is analog, so input is given to analog pin A0. Now, perform the AND operation on both the inputs and digital output is taken from digital pin 13. If the output is 1 i.e. high, the system allows the commuter to enter the building. This is depicted as LED glowing in the circuit. If the output is 0, i.e., low, the system does not allow the commuter to enter the building as he is either not wearing the mask or his body temperature exceeds normal human body temperature.

\section{VI.RESULT}

Computer Vision Unit: Camera equipped to the unit is able to detect the faces with and without the mask efficiently. The unit is well trained with the data set and is able to perform the task of detecting masks on the live video input[7]. When the person in front of the camera is wearing the mask, a green colored box appears around the face stating that person is wearing a mask. Unit gives out the digital output 1in this case. If the person is not wearing the mask, a red box appears around the face and digital output 0 is given out[5].

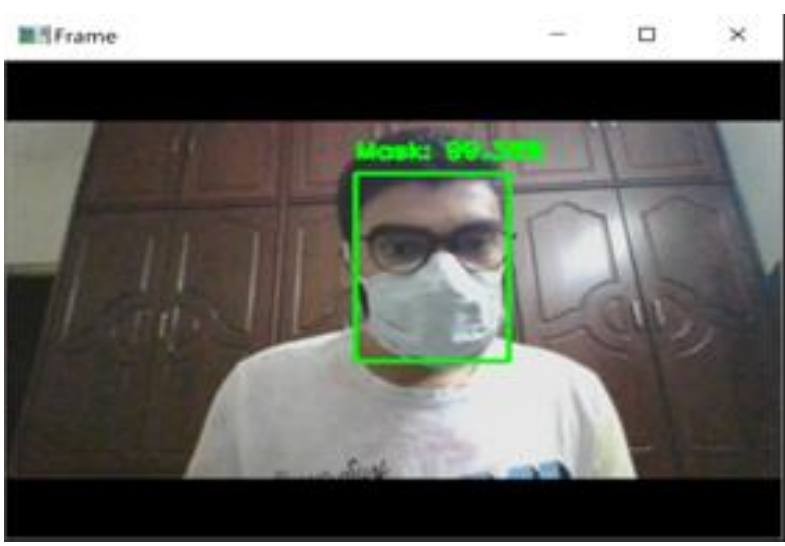

Fig. 4. Output of the unit for person without mask.

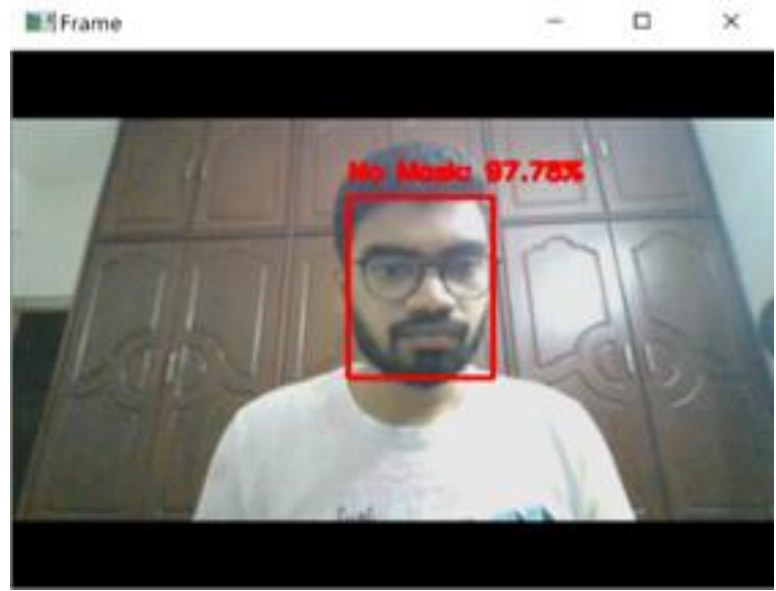

Fig. 5. Output of the unit for person with a mask.

A. Integrated Unit

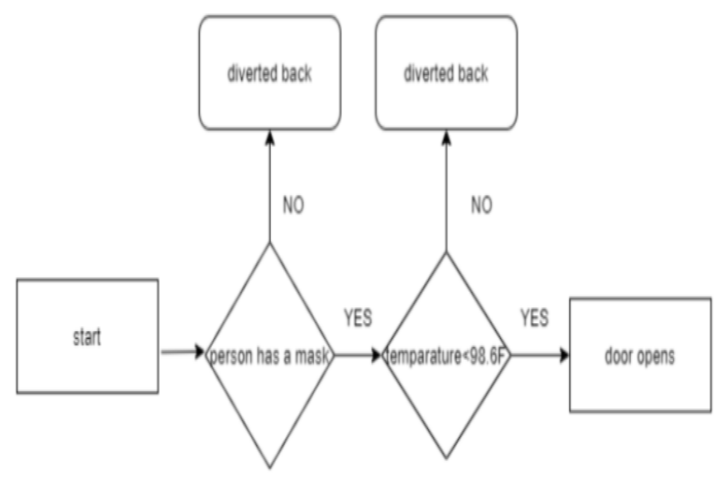

Fig. 6. Flowchart of the process.

Condition 1: When a commuter is not wearing a mask or has body temperature above $98.6 \mathrm{~F}$.

Table-I: Truth table of the above case

\begin{tabular}{|c|c|c|}
\hline Temperature & Mask & Output \\
\hline 0 & 0 & 0 \\
\hline 0 & 1 & 0 \\
\hline 1 & 0 & 0 \\
\hline
\end{tabular}

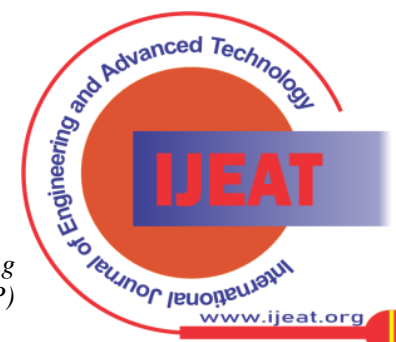




\section{Automated Screening System for Covid Safety}

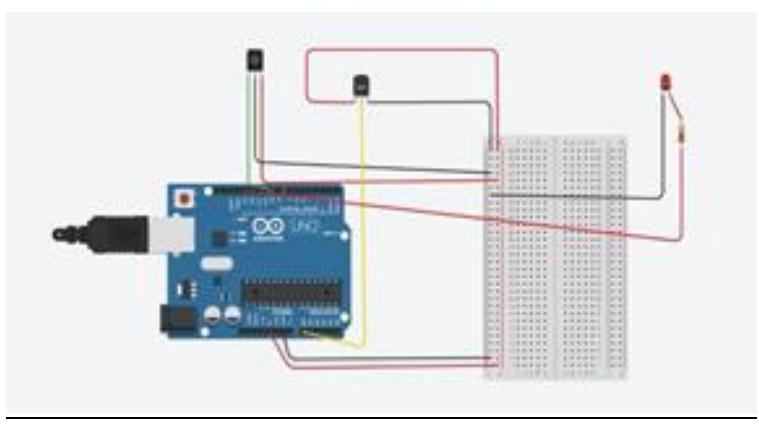

Fig. 7. Circuit implementation (LED is OFF)

Condition 2: When a commuter is wearing a mask and has body temperature below $98.6 \mathrm{~F}$.

Table-II: Truth table of the above case

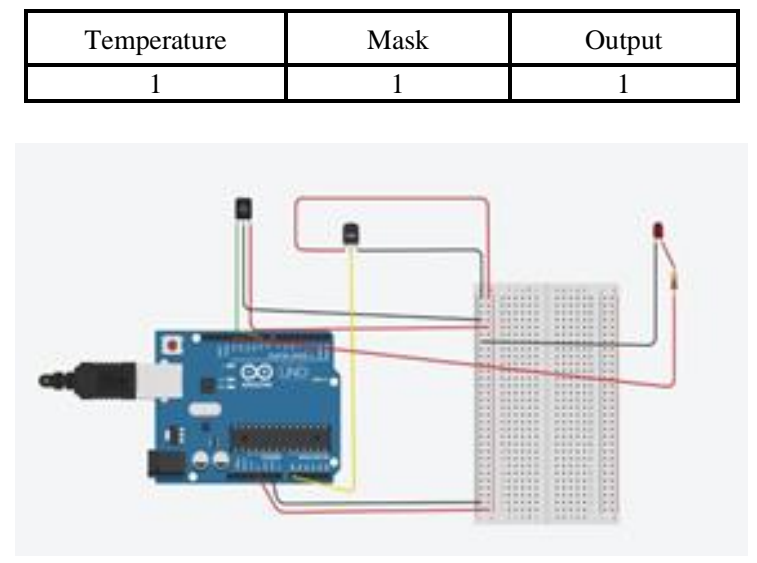

Fig. 8. Circuit implementation (LED is ON)

\section{CONCLUSION AND FUTURE SCOPE}

Individual units were designed effectively. The door, which is connected to the output of the integrated system, opens when a person, having normal body temperature, stands in front of the door wearing a mask. A person adhering to neither of the conditions when stands in front of the door, it will remain shut. Efficacy of the automated system for COVID safety can thus be observed. Sanitizer, a fundamental safety precaution can be included in the system. Commuters can be instructed to sanitize their hands by placing them under a dispenser which dispenses sanitizer by detecting hands using IR sensor and servo motor to open and close the lid of the dispenser. A mobile application can be made which can be used by the security personnel to get them notified about the individuals who try to enter the place without fulfilling the precautionary measures and they can intervene when their attention is required. Social distancing can also be monitored by using the computer vision techniques and openCV implemented by Raspberry Pi.

\section{REFERENCES}

1. Coronavirus

Update

[online]. https://www.worldometers.info/coronavirus/

2. L. Morawska, "How can airborne transmission of COVID-19 indoors be minimised?", Environment International vol. 142, September 2020, 105832, pp. 1-7, 2020. https://doi.org/10.1016/j.envint.2020.105832

3. D. Oran, E. Topol, Prevalence of Asymptomatic SARS-CoV-2 Infection:A Narrative Review, Annals of Internal Medicine, June 2020, pp. 1-7, 2020. https://doi.org/10.7326/M20-3012

4. Open Computer Vision [Online]. https://opencv.org/

5. P. Viola, M. Jones, "Rapid Object Detection using a Boosted Cascade of Simple Features", 2001 IEEE Computer Society Conference on Computer Vision and Pattern Recognition, pp. 511-518, 2001.

6. Raspberry Pi information, https://www.raspberrypi.org

7. Raspberry Pi camera module documentation, https://www.raspberrypi.org/documentation/hardware/camera/

8. TensorFlow documentation, https://www.tensorflow.org/api_docs

9. MobileNets: Efficient Convolutional Neural Networks for Mobile Vision Applications, https://keras.io/api/applications/mobilenet/

10. Convolutional Neural Network (CNN), https://www.tensorflow.org/tutorials/images/cnn

\section{AUTHORS PROFILE}

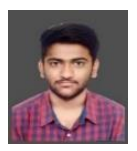

(3)

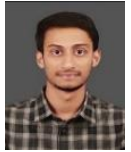

Y Sai Subhash Reddy, pursued his Bachelor of Technology in computer science engineering from Vellore Institute of Technology, Vellore. Working as Software Engineer at Bank of America.

Sri Krishna Borra, pursued his Bachelor of Technology in electronics and communication engineering from National Institute of Technology, Jamshedpur.

Koye Sai Vishnu Vamsi, pursued his Bachelor of Technology in computer science engineering from Vellore Institute of Technology, Vellore. Working as Software Engineer at Tata Consultancy services.

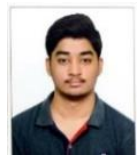

Nandipati Jaswanth Sai, pursued his Bachelor of Technology in computer science engineering from Vellore Institute of Technology, Vellore.

Koye Jayanth, pursuing his Bachelor of Technology in computer science engineering from Vellore Institute of Technology, Andhra Pradesh.

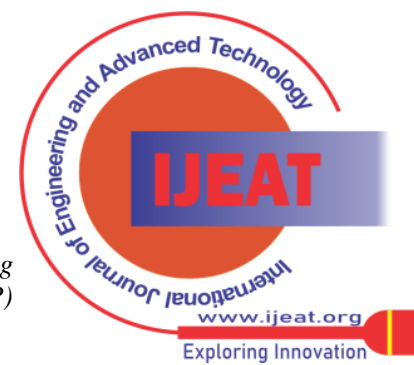

